\title{
Context-Aware Multi-Agent Systems: Negotiating Intensive Fields
}

\author{
> Rodrigo Shiordia López \\ University of Southern California, United States \\ rodrigo.shiordia@gmail.com
}

\author{
> Dr. David Jason Gerber \\ University of Southern California, United States \\ dgerber@usc.edu
}

\begin{abstract}
This paper presents research into a technique using context-aware agent based branching L-systems to design explore an urban development scheme in an area of peripheral Mexico City. The design research demonstrates a viable approach to engaging design with specific agent driven objectives that negotiate across highly differentiated fields of data sets. These data sets are the driving force behind this technique, to generate highly differentiated infrastructure and urban networks that are simulated to be autonomous and emergent. The described system consists of simulated robotic autonomous agents that sample and negotiate across data from the site, and react to differences in order to deploy an irrigation network for a polluted and highly saline former lake-bed east of Mexico City.
\end{abstract}

Reywords: Multi-Agent Systems; L-Systems, Generative Urban Design; Multi-Objective Optimization: Design Agency.

\section{Introduction}

This work situates itself amongst research that studies the importance of design exploration, parametric design thinking and specifically the development and use of multi-agent systems in architectural and urban design.(Gerber 2007) These systems are normally seen as bottom-up strategies that provide designers with novel ways to simulate complex relationships that they would normally would not be able to produce (Snooks 2013). The work consists of the development of a multi-agent system that organizes the territory based on data measured on site by a community of simulated robotic agents with a high degree of autonomy. We first establish the theoretical framework behind the design of the system, exploring the possible overarching principles behind its genesis. We then describe the proposed system through the design objectives, constraints, variables, and parameters, and then we evaluate some initial results. The evaluation of the performance of the system is performed by comparing different initial data sets with the final form of the irrigation network deployed in the simulation.

These results illustrate a viable workflow for negotiating with intensive fields generally, and more specifically provides us as architectural and urban designers a site-specific scheme for deployment of a highly intelligent and emergent infrastructure network for the purpose of exploring the potential of a novel concept of urban fertility. The work is evaluated in the context of generative urban design literature and discourse, for the ways in which architects are appropriating simulation technologies to generate urban form. This technique adds to the discourse a viable solution, one that breaks away from conventional tessellation driven solutions that are proliferating in academic environments.

\section{Background and Review}

Multi-Agent systems have been applied to design problems of varying scales ranging from urban design (Bus 2012, Popov 2011), to surface panelization (Baharlou and Menges 2013) or for materially or aesthetically driven architectures such as Snooks to name but a few (Snooks 2012). The swarm as a model for bottom up formation was described first for the computer vision and graphics domain and is now becoming prevalent within the architectural discourse (Reynolds 1987). Designer centric implementations of Reynolds' algorithm have become models for the evolution of systems that create geometry from many local interactions, both between elements in a system and between their environments (Schmidt 2012, Shiffman 2012, Aranda and Lasch 2006).

Simulation technologies including agent based design system are appropriated by architects into their design phase in order to improve the performance of the design at an early stage. While these technologies are very useful in simulating daylight, structural performance, fluid flows for example less attention has been placed into incorporating the simulation of pollution as a design driver (Weinstock and Stathopoulos 2006). Data from simulation has been used as a final validation of a design solution (Doumpioti 2010), or as an evaluation tool to compare large solution spaces from Genetic Algorithms and automated Multi-objective Design Optimization (Lin and Gerber 2014). Simulation data as a driver 
of design has also been explored with multi-agent swarms in order to generate material organization (Tsiliakos 2012, Gerber et al. 2014). Our work adds to this body of design research in a way that conceptualizes the swarm as an actual physical swarm, where the tasks performed in the simulation, simulate the tasks performed in the actual physical environment, where high degrees of noise and differentiation must be accommodated for and by the swarm of autonomous robotic agents. As such, the constraints of the technology and of the site's intrinsically intensive field conditions influence the design of the system.

There are precedent examples of programming robotic agents that are used for construction (Willmann, Federico Augugliaro, and et al. 2012, Leach 2009). In concert with these precedents our research conceptualizes a future where the technology will permit the deployment of autonomous robotic agents for large landscape urbanism scale implementations. Furthermore, our work breaks away from the notion that a collective of robotic agents should be programmed with a pre-set form, and thus eradicates a level of determinism for the designer to have to imagine form a priori as a crucial step for design. The paper presents a framework where the robot community are programmed the steps to perform in order to engage with the complexities of a real world site, and react to their local singularities without the need for the designer to preconceive the form.

L-Systems as an architectural and morphological logic have also been explored as a bottom-up system of generating urban and architectural form (Broughton and Coates 1997, Serrato-Combe 2005). These type of formal systems were similarly to the swarm or flock first explored in a domain different from design and architecture, that of cellular biology (Lindenmayer 1968). Here the system operates as a discrete version of the swarm, bottom-up in nature, providing a final output resulting from incessant local interactions in the context of highly gradient field condition.

\section{Methodology}

The research methodology is developed in consideration of a few principles that would guide the research; namely that of the need to engage highly differentiated field of intensities, with bottom up autonomy as a means to simulate new landscape urbanism, and finally that of the use of complex negotiation across and among agents as evidence for harnessing the design and meta heuristic value of multi-agent systems.

\section{Intensive fields}

We conceptualized the site as a field of intensities where any point in the site can be represented as an $\mathrm{n}$-dimensional vector including position $(x, y$, and $z$ ), and values representing a measure of a particular intensity. These intensities are conceptualized as generalizable but here included pollution levels, soil temperature and salinity. Intensive properties are represented as gradient maps, and only understood as the difference or variation from one point to the other (De Landa 2011). Thus the term intensive field describes a design space where there are several gradients super-imposed, and the varying intensities generate local differences on a site. The site as a field is an idea theoretically explored where the space is no longer a discrete array of unrelated properties, but rather a field of differentiated and inter-related intensities and capacities (Allen 1997). Our methodology uses the site's properties as relevant descriptors and drivers of agency, and to then choose properties that are operated upon as gradients for our system to interact with.

\section{Relevant descriptors}

A relevant descriptor is a property that is at the forefront of a system's behavior and performance (Poletto and Pasquero 2012). We borrowed this term as a way for us to extract relevant information from the site for use as an input gradient for our system of agents. In the case of this site, a former lake bed in Mexico City, elevation was rather constant, and thus not very relevant to the site, as we discovered in the research. On the other hand, we found that pollution and salinity patterns in the soil, and water table depths were in fact relevant as to how the site behaves, and how we could design explore rejuvenation and urban fertility. Plant populations and communities in the site respond more directly to these properties than to others. The variation of these intensive properties across the site was evident when looking at the gradients resulting from these properties (Anaya Rodríguez 1995, Coras-Merino 2006).

\section{Objective(s) and optimizations}

The initial design brief sampled and delineated across a largescale urban regeneration field that is highly data intensive and therefore complex to optimize in a purely top down fashion. It is important to note the boundaries of the design project, its scale and its internal highly differentiated topographical and pollutant intensities add levels of complexity difficult if not impossible for a human designer to sample, predict, and direct performance outcomes and optimality with high degrees of certainty. Intrinsic to our line of inquiry is one that is in part predicated on previous design computing and cognition research that suggests that the human designer is limited by issues of problem scale and coupling. Our observations, simplified, are that the human designer should be given more automation in order to aid in the management and production of ever more complex geometries while maintaining or enhancing their performance characteristics. (Flager, Gerber, and Kallman 2014) Another line of inquiry that is precedent to the work is that of methods that design explore and simultaneously optimize across multiple competing objectives seen in the previous work by the author (Gerber and Lin 2013). So the objectives are to provide efficiencies for designer through better tools described below, better ability to design with complexity, and improved ability to optimize complex design solutions where intensive fields of data are drivers.

\section{The System}

The workflow has been built around a series of open source simulation environments, an easy to use associative parametric design and visual programming environment Grasshopper/Rhinoceros and a series of plug-ins for integrating performance simulations and generating data that can then be read by a custom agent based 


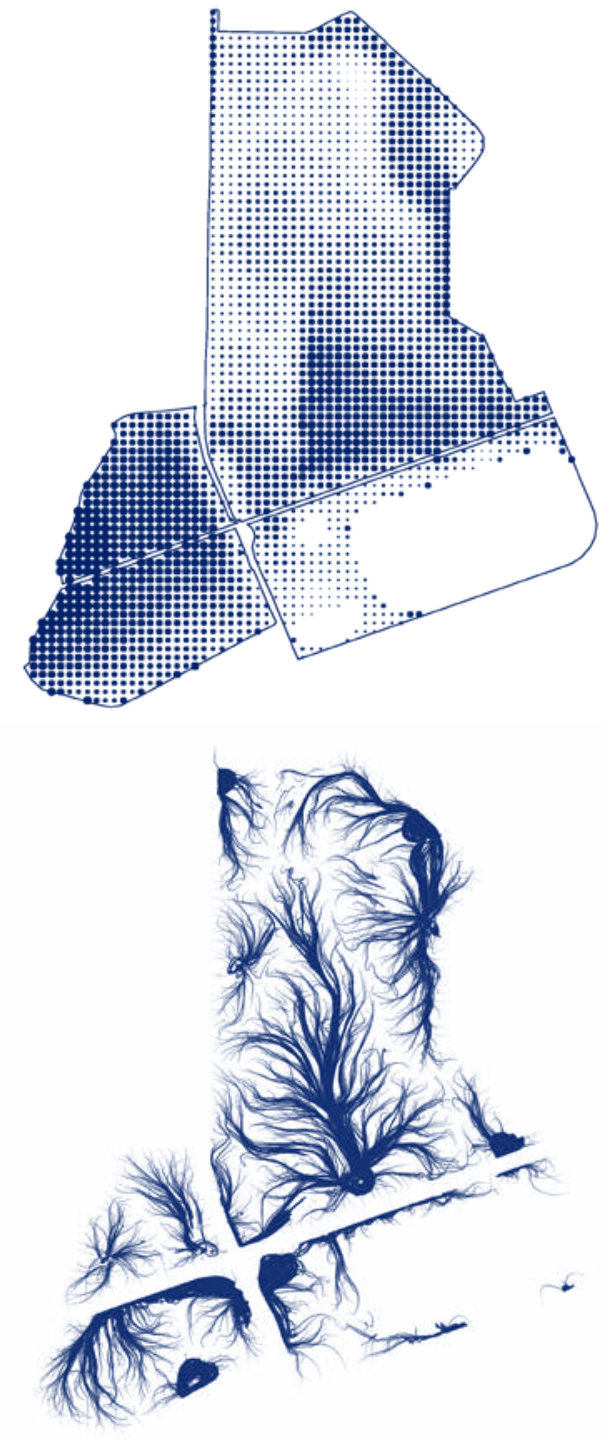

Figures 1 and 2: Illustrate the site and an approximation of the intensive field condition of soil salinity as a grid of points and magnitudes represented as diameters (figure 1), The bottom image (figure 2) illustrates a collective of agent paths deployed at every grid point where their trajectories are shown to illustrate the convergence of local maxima, reaction to themselves, to the boundary conditions of the site and the intensity of the field.

swarm algorithm programmed in the Processing 2.0 environment and language.(Sanchez 2013) The simulation data is exported as text files into our Processing sketch where the agents read the surface vertices and coupled data values. The agents are then spawned and are programmed to make movement and trajectory decisions based on the local information including the intensity values from the simulation, proximity to neighbors, and trails left by other agents. The multi agent system is based on flocking behaviors weighted by the data and recursive response to the overall dynamics. Each agent has the capacity to read the data from the simulation, which is paired with its corresponding point in a mesh object. Thus, the agent's environment is a collection of points to which it is constrained, and each point is assigned an intensity score based on the data from the simulation. The agent's trajectories then become a generative geometry for hypothetical robotic deposition of an irrigation network and for material re-organization. This happens in a collective recursion. We found that the sensing and deposition logic of the robot's field sampling and measuring had to be discretized in order to be feasible.

Through experimentation our first approach proved to be a very impractical sensing logic. It assumed that every agent knew the score of several hundred points at a time. There is no salinity sensor that can effectively do this practically speaking. Another issue is the fact that the field of view is actually limited to a couple of hundred feet wide, making it impossible for a sensor to be at all the sample points at any given time not withstanding they are yet to be determined. A second method was therefore proposed to take into consideration a discrete sensing of the space, making it practical for the agent to take one measurement at a time. This method simply changed the system's logic from one of swarm like behavior to a branching agent logic influenced by L-Systems. Branching structures have been studied in architecture for structural capabilities as in Frei Otto's work (Werkbund and Museum Villa 1995) and also for their fractal properties (Chan and Chiu 2000) (Hanafin, Datta, and Rolfe 2011). This new method specified a base, a sensing sequence that samples three points relative to the base, and a new base on the point with the highest intensity. It then repeats the process. This is a context or environment aware branching agent L-system, a very different application than what it has been used in the past. In this case,

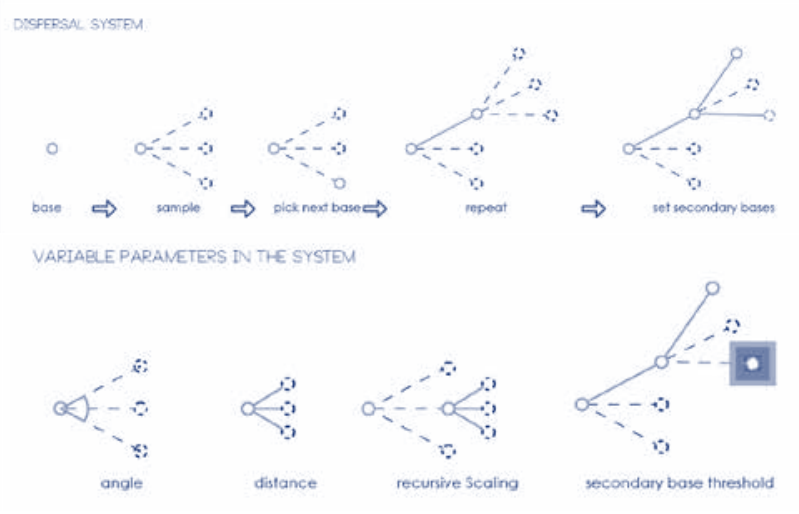

ADDITIONAL CONSTRAINTS
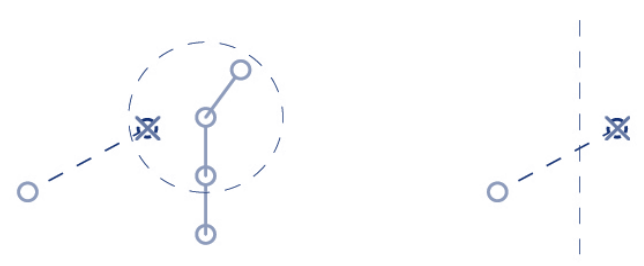

proximity to other dropped units

site boundary restriction

Figures $\exists$ through 5: show the pseudo-code of the second approach. The branching agent $\mathrm{L}$-system is based on a starting base, and then 3 relative points are measured in salinity. 
the point at which our system would select a new base would be the point with the lowest salinity of the three sampled points.

Our current focus and input gradient is that of soil salinity because we found techniques of reducing soil salinity by way of irrigation for defining our landscape urbanism. These techniques are common throughout agriculture as a way of cultivating land with high levels of salinity, the technique is known as strip cropping, and consists of the alternative irrigation of strips of land, leaving fallow strips in between. Water will travel from the irrigated strips to the fallow strips, leeching and dissolving salts, which accumulate through seepage on the fallow strips. We hypothesized that if we could generate a system of autonomous robotic agents to deploy an irrigation network in the parts of the site with lower relative salinity, these tracts would gradually become less saline, leaving ground for re-cultivation and encapsulating areas for urban densification.
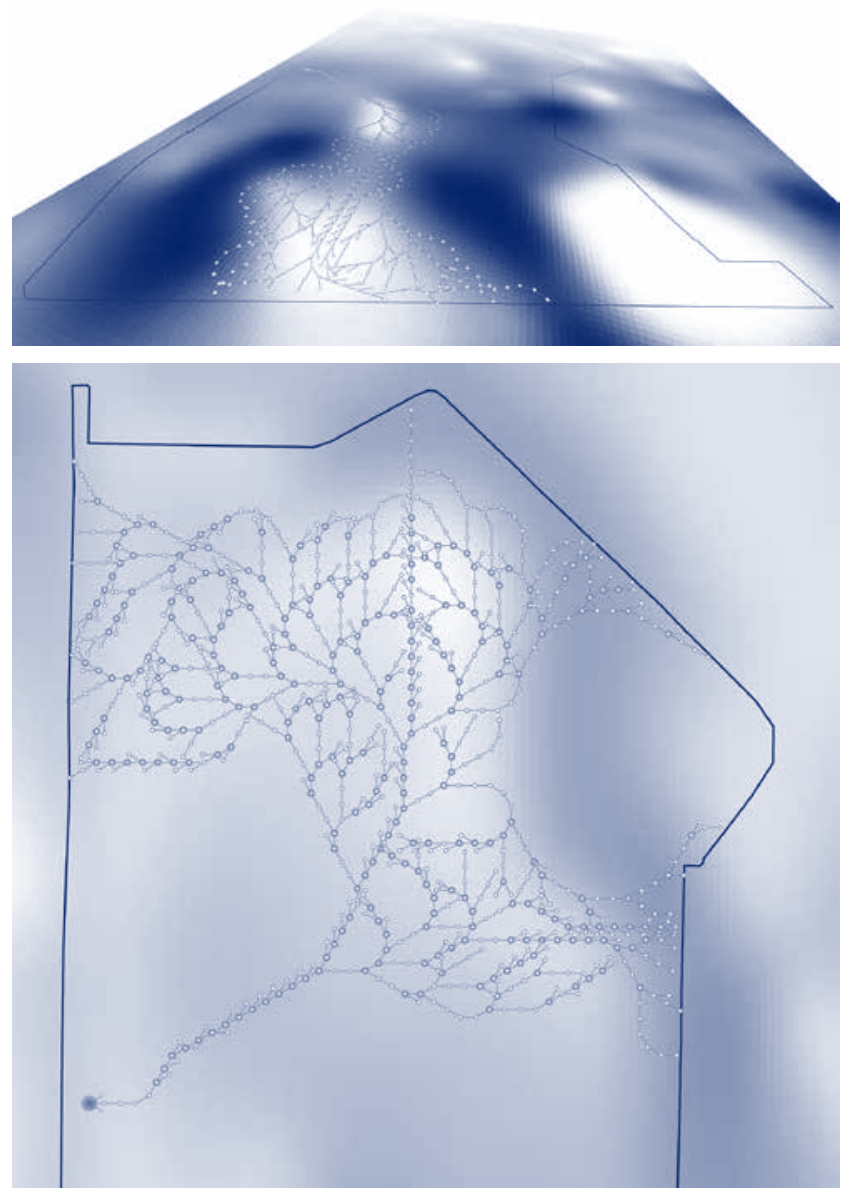

Figures 6 and 7: show the resulting network drawn based on the gradient field (left), a superimposed dataset and its corresponding network (middle), and the irrigation network as a basis for urban development with finally the new gradient calculated by proximity to the network. We can see that the irrigation network accumulates in lighter areas, and avoids darker areas. This was our objective, however, we did not purposefully place any point except the starting point in the network.

\section{Urban Fertility}

As a way to propose urban growth, the concept of urban fertility was coined in order to capture our design goal of urban and landscape rejuvenation. Once we had the irrigation network deployed on the site, we could assume that those areas would in fact become less saline over time, and the more saline areas would be around the irrigation network, it is these latter areas where the city infrastructure could more easily exist on site without interfering with the soil remediation and cultivation processes. Thus, these areas were deemed to have more urban fertility than others.

\section{Conclusions}

The resulting urban scheme, while still at a conceptual level, experiments upon the notion that city growth, as a bottom up and emergent approach can be autonomously derived through multi-agent based design systems. It is further experimented upon through simulation of a robotic community of agents with in-built objectives to support urban densification while negotiating with needs for urban ecology and agriculture. These designer bots in effect a community of agents programmed to populate, disperse, connect and pattern through branching and l-system like behaviors, are designed through an environmental performance in constant local and global negotiation with themselves and across a multiplicity of theorized sensor based intensities in which to re-purpose and redefine the possibilities of a forgotten and polluted lake bed for Mexico city. On top of the design research we also analzyzed the results with empiricism to conjecture further developments of the system and for further test beds.

One measured result is that the system did in fact populate areas with lower salinity with the irrigation infrastructure, while the darker areas were in fact populated with urban masses. Initially his resulted in a 40-60 scheme of land reserved for natural regeneration (40\%) and

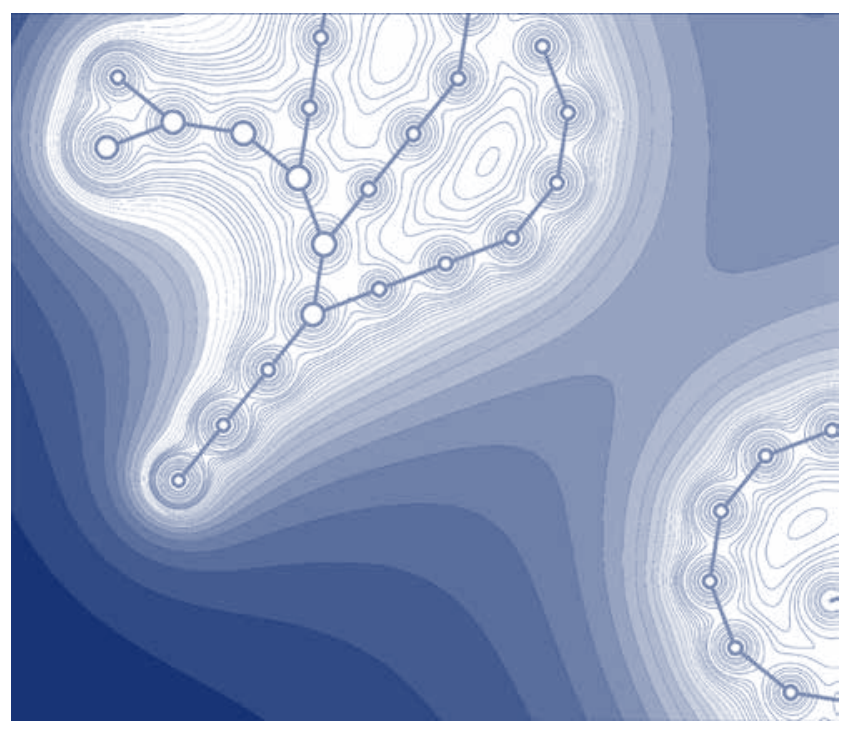

Figures 8: shows close-up of the system and a possible configuration of urban fertility, the irrigation network deployed, and the resulting gradient representing urban fertility. 


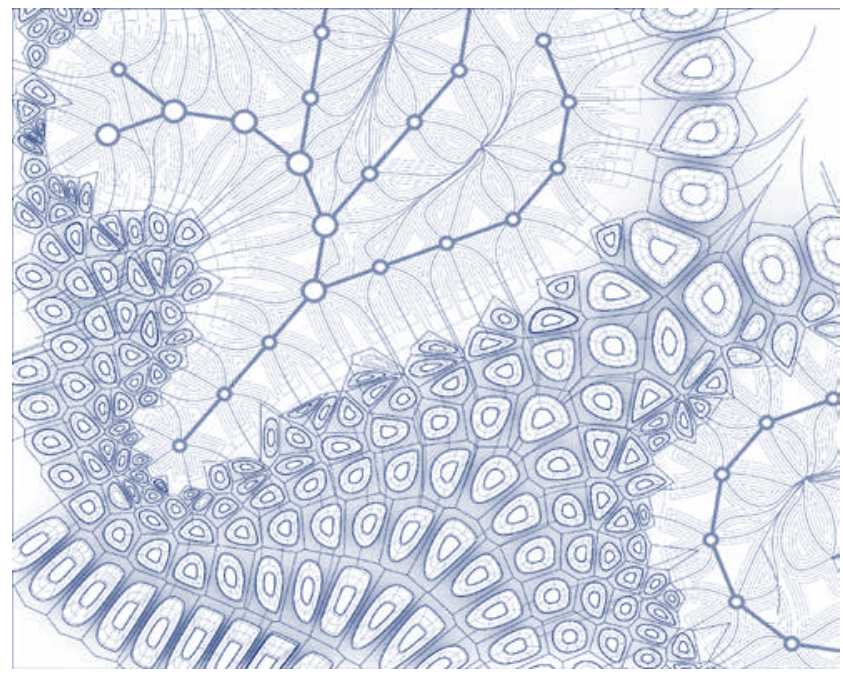

Figure 9: is a plan view illustrating the resultant urban massing and densification pattern where darker areas from Figure 8 are populated with larger virtual masses.

land used for urban structures (60\%). We did not expect to have such a proportion, and this is a generatively designed result. Taking into consideration the fact that we only used one starting point in these examples, we can conjecture that the system is effective in generating a differentiated field of landscape and urban massing. The evaluation is that of actual floor area where we had 6,329,567 square meters of land targeted for landscape remediation, and 10,232,753 square meters for urban development. Even though we were not constrained to a specific ratio, the fact that this proportion was reached descriptively suggests that the system is not a stochastic or pseudo-random process. Future work will include testing and developing more realistic and measured the systems variables (spawning points, angles, distances, etc) in order to attain specific proportions and more informed objectives for our contextually aware multi- agent driven branching system that negotiate the complexities of real world intensive fields.

\section{Acknowledgments}

We would like to thank Alvin Huang, and Jose Sanchez for their guidance and advice in the process of pursuing this research. We would also like to acknowledge and thank the USC School of Architecture Dean, Qingyun Ma, for his support of the work through the Graduate Research Student funding program.

\section{References}

Allen, Stanley. 1997. "From object to field." Architectural design no. 67 (5-6):24-31.

Anaya Rodríguez, Edgar. 1995. "The Industry of Earth Salt in the Valley of Mexico: A prehispanic method in the verge of disappearing." In Salt in Mexico, edited by Juan Carlos Reyes, 223-248. University of Colima, General Bureau of Popular Cultures-CNCA,

Aranda, Benjamin, and Chris Lasch. 2006. "Flocking." In Tooling. New York: Princeton Architectural Press.

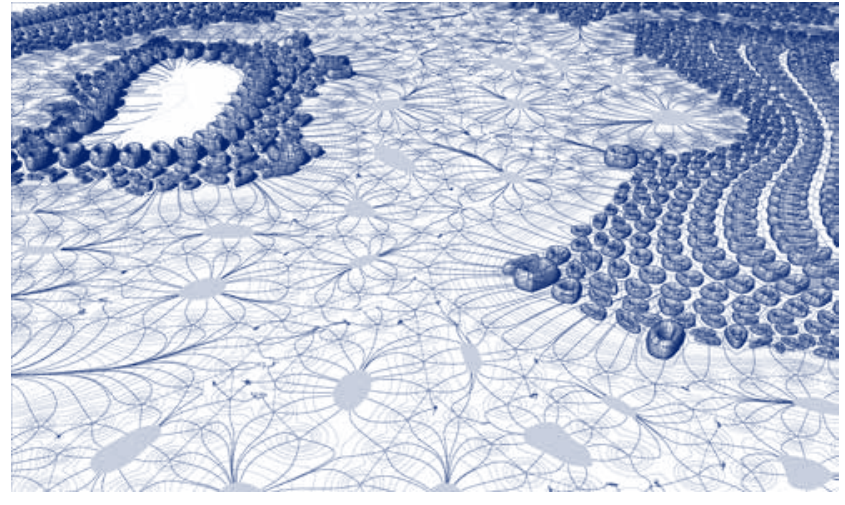

Figure 10: a perspective view illustrating a possible urban massing and densification pattern and irrigation network.

Baharlou, Ehsan, and Achim Menges. 2013. Generative AgentBased Design Computation. In Computation and Performance - Proceedings of the 31st eCAADe Conference edited by Rudi and Sariyildiz Stouffs, Sevil (eds.). Faculty of Architecture, Delft University of Technology, Delft, The Netherlands, : eCAADe

Broughton, T., Tan, A., and P.S. Coates. 1997. The Use of Genetic Programming In Exploring 3D Design Worlds - A Report of Two Projects by Msc Students at CECA UEL, CAAD Futures 1997 [Conference Proceedings / ISBN 0-7923-4726-9] München (Germany), 4-6 August 1997, pp. 885-915.

Bus, Peter. 2012. Emergence as a Design Strategy in Urban Development: Using Agent-Oriented Modelling in Simulation of Reconfiguration of the Urban Structure. In Digital Physicality - Proceedings of the 30th eCAADe Conference, edited by Henri; Pavlicek Achten, Jiri; Hulin, Jaroslav; Matejdan, Dana (eds.). Czech Technical University in Prague, Faculty of Architecture (Czech Republic)

Coras-Merino, Pablo Miguel. 2006. "Climatic Elements and their relation to fluctuations on water table depths." TERRA Latin American no. 24 (3, July-September):375-382.

Chan, Chen-Wei, and Mao-Lin Chiu. 2000. A Simulation Study of Urban Growth Patterns with Fractal Geometry. In CAADRIA 2000 [Proceedings of the Fifth Conference on Computer Aided Architectural Design Research in Asia I ISBN 981-04-2491-4] Singapore 18-19 May 2000, pp. 55-64.

De Landa, Manuel. 2011. Philosophy and simulation: the emergence of synthetic reason. London;New York: Continuum.

Doumpioti, Christina. 2010. Fibre composite systems: stress as growth-promoting agent. In Proceedings of the 15th International Conference on Computer Aided Architectural Design Research in Asia.

Flager, Forest, David Jason Gerber, and Ben Kallman. 2014. "Measuring the impact of scale and coupling on solution quality for building design problems." Design Studies no. 35 (2):180-199. doi: http://dx.doi.org/10.1016/j. destud.2013.11.001. 
Gerber, David Jason. 2007. Parametric practices: Models for design exploration in architecture. Dissertation, Architecture, Harvard Graduate School of Design, Cambridge, MA.

Gerber, David Jason, and Shih-Hsin Eve Lin. 2013. "Designing in complexity: Simulation, integration, and multidisciplinary design optimization for architecture." Simulation no. Published online before print April 9, 2013. doi: 10.1177/0037549713482027.

Gerber, David, Rodrigo Shiordia, Sreerag Veetil, and Arjun Mahesh. 2014. Design Agency: Prototyping Multi-Agent System Simulation for Design Search and Exploration. In 2014 Proceedings of the Symposium on Simulation for Architecture and Urban Design edited by David and Goldstein Gerber, Rhys. Tampa, FL, USA: SIMULATION COUNCILS, INC.

Hanafin, Stuart, Sambit Datta, and Bernard Rolfe. 2011. Tree facades: Generative modelling with an axial branch rewriting system. In Proceedings of the 16th International Conference on Computer Aided Architectural Design Research in Asia / The University of Newcastle, Australia 27-29 April 2011, pp. 175-184.

Leach, Neil. 2009. "Swarm Urbanism." Architectural Design no. 79 (4):56-63. doi: 10.1002/ad.918.

Lin, Shih-Hsin Eve, and David Jason Gerber. 2014. "Designing-in performance: A framework for evolutionary energy performance feedback in early stage design." Automation in Construction no. 38:59-73. doi: 10.1016/j.autcon.2013.10.007.

Lindenmayer, Astrid. 1968. "Mathematical models for cellular interaction in development." Theoret. Biology no. 18:280—315.

Poletto, Marco, and Claudia Pasquero. 2012. Systemic architecture: operating manual for the self-organizing city. Abingdon, Oxon [England] ;New York, NY: Routledge.

Popov, Nikolay. 2011. Generative sub-division morphogenesis with Cellular Automata and Agent-Based Modelling, RESPECTING FRAGILE PLACES [29th eCAADe Conference Proceedings / ISBN 978-9-4912070-1-3], University of Ljubljana, Faculty of Architecture (Slovenia) 21-24 September 2011, pp.166-174.

Reynolds, Craig. 1987. "Flocks, Herds, and Schools:

A Distributed Behavioral Model." Computer Graphics no. 21 (4, July 1987):25-34.
Sanchez, Jose. 2013. Plethora, A Library for the Programming Environment Processing Jose Sanchez.

Schmidt, Karsten. 2012. Toxiclibs, Building Blocks for Computational Design.

Serrato-Combe, Antonio. 2005. Lindenmayer Systems - Experimenting with Software String Rewriting as an Assist to the Study and Generation of Architectural Form, SIGraDi 2005 - [Proceedings of the 9th Iberoamerican Congress of Digital Graphics] Lima - Peru 21-24 november 2005, vol. 1, pp. 161-166.

Shiffman, Daniel. 2012. The Nature of Code. Simulating Natural Systems with Processing.

Snooks, Roland. 2013. Snooks, Roland. Fibrous Assemblages and Behavioral Composites, The Funambulist. Accessed July 25th. 2012 [cited July 25th 2013 2013]. Available from http://thefunambulist.net/2012/04/20/guest-writers-essays-25-fibrous-assemblages-and-behavioral-composites-by-roland-snooks/.

Snooks, Roland. 2013. "Behavioral Formation \& Material Assemblages." In Fresh Punches: Experimental Architecture Exhibition Catalogue, edited by Nathan Hume, et. al.

Tsiliakos, Marios. 2012. Swarm Materiality: A multi-agent approach to stress driven material organization. In Digital Physicality Proceedings of the 30th eCAADe Conference.

Weinstock, Michael, and Nikolaos Stathopoulos. 2006. "Advanced simulation in design.” Architectural Design no. 76 (2):54-59. doi: 10.1002/ad.240.

Werkbund, Bayern, and Stuck Museum Villa. 1995. Frei Otto, Bodo Rasch: finding form: towards an architecture of the minimal: the Werkbund shows Frei Otto, Frei Otto shows Bodo Rasch : exhibition in the Villa Stuck, Munich, on the occasion of the award of the 1992 Deutscher Werkbund Bayern prize to Frei Otto and Bodo Rasch. Stuttgart?: Axel Menges.

Willmann, Jan, Thomas Cadalbert Federico Augugliaro, ., and et al. 2012. "Aerial Robotic Construction Towards a New Field of Architectural Research." International Journal of Architectural Computing no. 10 (3):439-459. 